\title{
Investigação epidemiológica da brucelose bovina em um estrato do Estado de Mato Grosso do Sul ${ }^{1}$
}

\author{
Letícia Almeida R.C. Monteiro ${ }^{1 *}$, Aiesca O. Pellegrin ${ }^{2}$, Márcia M. Ishikawa ${ }^{3}$ e Ana \\ Luiza A.R. Osório ${ }^{4}$
}

\begin{abstract}
Monteiro L.A.R.C., Pellegrin A.O., Ishikawa M.M. \& Osório A.L.A.R. 2006. [Epidemiological investigation of bovine brucellosis in an extract of Mato Grosso do Sul, Brazil.] Investigação epidemiológica da brucelose bovina em um estrato do Estado de Mato Grosso do Sul. Pesquisa Veterinária Brasileira 26(4):217-222. Programa Mestrado em Ciência Animal, Universidade Federal do Mato Grosso do Sul, Campo Grande, MS 79070-900, Brazil. E-mail: leticiacmonteiro@yahoo.com.br

The study aimed to estimate the prevalence of bovine brucellosis in 22 counties which make up the region Extract 1 of the State of Mato Grosso do Sul, Brazil, in order to identify risk factors associated with the infection. The sample region encompasses an area of $70,214.1 \mathrm{~km}^{2}$ and represents $19.7 \%$ of the State. The region studied has about 5.7 million head of cattle, corresponding to $23 \%$ of the total of 24.9 million cattle in the State of Mato Grosso do Sul. On 210 farms, between December 2003 and March 2004, 2,376 blood samples were collected from cows, aged 24 months or older, for serial diagnostic tests. Screening through the buffered acidified antigen test was confirmed by the 2-mercaptoetanol test. On the occasion of sample collection a questionnaire with information related to identification, kind of cattle and management practices was filled out. In individual animals the real prevalence was estimated at $5.6 \%$, and in the cattle herds at $37.3 \%$. The variables, which presented association through odds ratio (OR), univariate analysis and 95\% confidence interval $(\mathrm{CI})$ with serum positivity for brucellosis, were: the exploration of beef cattle $(\mathrm{OR}=2.82,95 \% \mathrm{CI}=1.49-5.34)$, Zebu breed $(\mathrm{OR}=2.62,95 \% \mathrm{CI}=1.40-4.88)$ and abortion $(\mathrm{OR}=$ $1.83,95 \% \mathrm{CI}=1.01-3.33)$. The results shown here demonstrate, despite the prevalence of brucellosis in the extract of Mato Grosso do Sul studied, that the control of the disease may depend on adoption of a program focusing upon the exploration of beef cattle, the Zebu breed and the occurrence of abortion.
\end{abstract}

INDEX TERMS: Brucellosis, cattle, epidemiology, seroprevalence.

RESUMO.- O objetivo deste estudo foi estimar a prevalência da brucelose bovina nos 22 municípios que compõem a região denominada Estrato 1 do Estado de Mato Grosso do Sul, e identificar os fatores de risco associados à infecção. A região amostrada constitui uma área de $70.214,1 \mathrm{~km}^{2}$, que representa $19,7 \%$ do

\footnotetext{
1 Recebido em 11 de outubro de 2005.

Aceito para publicação em 10 de maio de 2006

1 Agência Estadual de Defesa Sanitária Animal e Vegetal (IAGRO/MS), Mestranda do Programa Ciência Animal da Universidade Federal de Mato Grosso do Sul (UFMS). Cidade Universitária, Campo Grande, MS 79070900. *Autor para correspondência: leticiacmonteiro@yahoo.com.br

2 Embrapa Pantanal, Cx.Postal 109, Corumbá, MS 79320-900.

3 Embrapa Agropecuária Oeste, Cx.Postal 661, Dourados, MS 79804970.

${ }^{4}$ Laboratório de Bacteriologia, Faculdade de Medicina Veterinária e Zootecnia, UFMS, Cidade Universitária, Campo Grande, MS 79070-900.
}

Estado. O rebanho de região estudada é de, aproximadamente, 5,7 milhões de cabeças, correspondente a $23 \%$ do efetivo de 24,9 milhões de bovinos de Mato Grosso do Sul. Nas 210 propriedades amostradas, no período de dezembro de 2003 a março de 2004, foram colhidas 2.376 amostras de sangue de fêmeas com idade igual ou superior a 24 meses, submetidas a testes diagnósticos em série. A triagem, realizada por meio do teste do antígeno acidificado tamponado, foi seguida pelo teste confirmatório 2-mercaptoetanol. Na mesma ocasião da colheita das amostras, foi preenchido um questionário com informações de identificação, tipo de criação e práticas de manejo. Em animais, a prevalência real foi estimada em $5,6 \%$, e em rebanhos, $37,3 \%$. As variáveis que apresentaram associação, por meio da análise univariada odds ratio (OR) e intervalo de confiança (IC) de $95 \%$, com a soropositividade à brucelose foram: o tipo de exploração corte $(\mathrm{OR}=2,82$, IC $95 \%=1,49-5,34)$, a raça Zebu $(O R=$ 
$2,62$, IC $95 \%=1,40-4,88)$ e o aborto $(O R=1,83$, IC $95 \%=1,01$ $3,33)$. Os resultados demonstram que, além da brucelose ser prevalente no estrato estudado em Mato Grosso do Sul, o controle da doença pode consistir na adoção de programa com especial atenção à exploração do tipo corte, à raça Zebu e à presença do aborto.

TERMOS DE INDEXAÇÃO: Brucelose, bovinos, epidemiologia, soroprevalência.

\section{INTRODUÇÃO}

O último diagnóstico nacional de situação da brucelose bovina foi realizado em 1975, tendo estimado a porcentagem de animais sororreatores em $4 \%$ na região Sul, $7,5 \%$ na Sudeste, $6,8 \%$ na Centro-Oeste, $2,5 \%$ na Nordeste e $4,1 \%$ na Norte (Poester et al. 2002, Brasil 2003).

Em Mato Grosso do Sul (MS), a prevalência estimada de brucelose em 1998 foi de 6,3\%, a mesma obtida em 1975, no então Estado do Mato Grosso (Paulin \& Ferreira Neto 2003). Outros estudos, adotando diferentes metodologias, realizados pela Universidade Federal de Mato Grosso do Sul, no período de 1982-1984 (Cavalléro 1998), em 2001 (Almeida 2001) e no Pantanal Sul-Mato-Grossense (Pellegrin et al. 1999), indicaram soroprevalências de $10,2 \%, 8,6 \%$ e $3,4 \%$, respectivamente.

Conforme boletins oficiais do Ministério da Agricultura, Pecuária e Abastecimento (MAPA), estudos realizados entre 1988 e 1998 indicaram que a prevalência de animais soropositivos para brucelose no Brasil variava entre 4 e 5\%, sugerindo esta doença como uma das principais causas de aborto em bovinos (Poester et al. 2002, Brasil 2003).

As perdas econômicas causadas pela brucelose são decorrentes dos sucessivos abortamentos e períodos de esterilidade temporária, responsáveis pela redução do desempenho reprodutivo dos rebanhos e baixa produção de leite (Campaña et al. 2003, Paulin \& Ferreira Neto 2003). No Brasil, os prejuízos econômicos ocasionados pela brucelose bovina ou bubalina foram estimados, em 1971 pelo MAPA, em US\$ 32 milhões de perdas anuais, considerando somente os abortos e a queda na produção leiteira (OIE 1987, Poester et al. 2002).

A transmissão da brucelose entre rebanhos deve-se à aquisição de animais infectados, proximidade a rebanhos infectados, que compartilham pastagem e água, e animais que se alimentam de outros em decomposição. Dentro do rebanho, a transmissão da enfermidade é influenciada pelo nível de vacinação, tamanho do rebanho, condições de instalações, uso de piquete-maternidade e densidade populacional (Crawford et al. 1990).

Com a implantação do Programa Nacional de Controle e Erradicaação da Brucelose e Tuberculose (PNCEBT) pelo MAPA (Brasil 2001), houve uma demanda para a execução de um inquérito soro-epidemiológico no Mato Grosso do Sul, visando a traçar o diagnóstico situacional da doença, servindo também como parâmetro de avaliação para o Programa.

Os objetivos deste estudo foram estimar a prevalência dos rebanhos bovinos infectados com Brucella abortus nos 22 municípios que compõem o Estrato 1 e levantar os principais fatores de risco relacionados com a ocorrência da doença nesses rebanhos.

\section{MATERIAL E MÉTODOS}

\section{Área de estudo}

Realizou-se o presente estudo na região denominada Estrato 1 do Estado de Mato Grosso do Sul (MS). Esse estrato, composto de 22 municípios (Quadro 1), faz parte da estratificação realizada pela coordenação estadual do PNCEBT, em quatro estratos distintos, com número de rebanhos equivalentes.

$\mathrm{O}$ estrato considerado abrange municípios pertencentes a três das quatro mesorregiões geográficas do Estado: centro-norte, leste e sudoeste (IBGE 2002), os quais possuem propriedades de extensão variada, dedicadas à exploração pecuária de corte e/ou leite e algumas desenvolvem atividade agrícola. Constitui uma área de $70.214,1 \mathrm{~km}^{2}$, que representa 19,7 \% de MS (IBGE 2002). O rebanho bovino da região estudada é de aproximadamente 5,7 milhões de cabeças, correspondentes a $23 \%$ do rebanho total do Estado, que é de 24,9 milhões (IAGRO 2003).

\section{Métodos de amostragem}

$\mathrm{O}$ desenho e métodos de amostragem foram baseados no inquérito soroepidemiológico da brucelose proposto pelo MAPA às unidades federativas, e a prevalência da brucelose foi estimada, tanto para rebanhos como para animais, sendo os resultados inferidos para o estrato em estudo.

Os rebanhos foram considerados as unidades primárias de amostragem. Para definir o tamanho da amostra, estimando uma proporção, considerou-se o grau de confiança de 95\%, o nível de precisão absoluta dos resultados de $5 \%$ e o valor da prevalência esperada ou mais provável de $16 \%$ (Noordhuizen et al.1997).

A amostragem resultou em 207 e decidiu-se por 210 propriedades (Quadro 1). No sorteio das propriedades foi utilizada a listagem das fichas sanitárias fornecidas pela Agência Estadual de Defesa Sanitária Animal e Vegetal (IAGRO), e para o cálculo do intervalo amostral dividiu-se o número total de propriedades pelo número de propriedades a serem amostradas. Em cada propriedade sorteada, o rebanho-alvo

Quadro 1. Rebanhos e bovinos do estrato do Estado de Mato Grosso do Sul estudados, amostrados para o diagnóstico de brucelose por município

\begin{tabular}{ccc}
\hline Município & $\begin{array}{c}\text { Rebanhos } \\
\text { amostrados }\end{array}$ & $\begin{array}{c}\text { Animais } \\
\text { amostrados }\end{array}$ \\
\hline
\end{tabular}

$\begin{array}{lcc}\text { Angélica } & 6 & 44 \\ \text { Bandeirantes } & 8 & 110 \\ \text { Caarapó } & 8 & 87 \\ \text { Campo Grande } & 18 & 206 \\ \text { Deodápolis } & 9 & 78 \\ \text { Douradina } & 2 & 20 \\ \text { Dourados } & 17 & 164 \\ \text { Fátima do Sul } & 5 & 36 \\ \text { Glória de Dourados } & 9 & 82 \\ \text { Itaporã } & 6 & 60 \\ \text { Ivinhema } & 14 & 134 \\ \text { Jaraguari } & 10 & 104 \\ \text { Jateí } & 7 & 76 \\ \text { Maracaju } & 10 & 140 \\ \text { Nova Alvorada do Sul } & 8 & 110 \\ \text { Novo Horizonte do Sul } & 10 & 125 \\ \text { Ribas do Rio Pardo } & 18 & 212 \\ \text { Rio Brilhante } & 6 & 80 \\ \text { Rochedo } & 6 & 78 \\ \text { Sidrolândia } & 17 & 235 \\ \text { Terenos } & 11 & 145 \\ \text { Vicentina } & 5 & 50 \\ \text { Totais } & 210 & 2.376\end{array}$


foi o de principal objetivo da produção e representado por animais nas mesmas condições de manejo.

Após o sorteio das propriedades procedeu-se ao sorteio dos animais, fêmeas, de idade igual ou superior a 24 meses, para serem sangradas. Em cada propriedade sorteada, onde existiam até 99 fêmeas na faixa etária igual ou superior a dois anos, foram amostradas dez fêmeas, ou todas as fêmeas dessa faixa etária, se elas fossem menos do que dez. Naquelas onde o número de fêmeas de idade igual ou superior a dois anos era superior a 99, foram amostradas 15 fêmeas dessa faixa etária.

A seleção das fêmeas sangradas foi feita de forma aleatória, empregando-se o método de amostragem aleatória simples ou sistemática. A escolha por um dos métodos foi definida dividindo o total de fêmeas com idade igual ou superior a dois anos existentes na propriedade pelo total de fêmeas a serem amostradas. Para resultados inferiores a dois, foi empregado o método de amostragem aleatória simples; nos casos em que o resultado era superior a dois, foi empregado o método de amostragem aleatória sistemática. O Quadro 1 sumariza os resultados dos cálculos realizados para escolha das propriedades amostradas e para a escolha das fêmeas que foram sangradas.

\section{Obtenção das amostras}

As amostras de sangue foram colhidas no período de dezembro de 2003 a março de 2004, por punção jugular, com agulha descartável, uma para cada animal e transportadas para o Laboratório de Diagnóstico de Doenças Animais (Laddan), da IAGRO. Os soros sanguíneos obtidos foram estocados a $-20^{\circ} \mathrm{C}$ e, depois, submetidos aos testes de diagnóstico de brucelose.

\section{Testes diagnósticos}

Para o diagnóstico foram empregados testes em série, visando a aumentar a especificidade (Thrusfield 1984). Como teste de triagem foi realizado o teste do antígeno acidificado tamponado (AAT), e os soros reagentes nesse foram submetidos ao teste confirmatório, 2mercaptoetanol (2-ME).

Os testes de AAT e 2-ME foram feitos no LADDAN, segundo protocolo preconizado pelo manual técnico do PNCEBT. No teste do AAT foi utilizado o antígeno na concentração de $8 \%$, tamponado em pH ácido $(3,65)$ e as bactérias coradas com rosa-de-bengala, e no teste do 2-ME foi utilizado o antígeno para prova de soroaglutinação lenta, com concentração de massa bacteriana de $4,5 \%$, sem corante, padronizado a partir da cepa 119-3 de Brucella abortus, inativado pelo calor, ambos os antígenos produzidos pelo Instituto de Tecnologia do Paraná (TECPAR).

Como os testes diagnósticos utilizados foram empregados em série, os cálculos de especificidade serial (EspS) e de sensibilidade serial (SenS) foram feitos de acordo com Noordhuizen et al. (1997). Os cálculos foram baseados, para o AAT, em Davies (1971), a partir dos valores de sensibilidade $(0,996)$ e de especificidade $(0,831)$. Para o 2-ME baseou-se em Vasconcellos et al. (1987), a partir da sensibilidade $(0,95)$ e especificidade $(0,92)$.

Os resultados dos testes diagnósticos foram positivos, negativos ou inconclusivos e classificou-se como positiva a propriedade que apresentou pelo menos um animal positivo no teste confirmatório (2-ME); negativa, quando todas as fêmeas apresentaram-se negativas nos testes sorológicos; e inconclusiva, quando existiram resultados sorológicos negativos e inconclusivo em pelo menos uma das fêmeas.

\section{Questionário}

De forma simultânea à colheita de sangue em cada propriedade, foram realizados entrevista e preenchimento de um questionário que incluía informações de identificação, tipo de criação, práticas de manejo, características relacionadas com as propriedades e registros sobre as amostras colhidas.

\section{Banco de dados}

Os resultados dos testes de diagnóstico e as informações do questionário aplicado em cada propriedade foram armazenados em um banco de dados, utilizando o programa Microsoft Access 97, desenvolvido para o PNCEBT.

\section{Análise estatística}

As prevalências aparente $(\mathrm{Pa})$ e real $(\mathrm{Pr})$ para animais bem como o intervalo de confiança (IC) foram calculados conforme Martin et al. (1987). A Pr foi estimada ajustando-se o valor de Pa obtida para a especificidade (Esp) e sensibilidade (Sen) dos testes diagnósticos (Martin et al. 1992).

A prevalência aparente de rebanho (PaR) e o IC foram calculados conforme Martin et al. (1987). A prevalência real de rebanho (PrR) foi estimada considerando-se a especificidade e a sensibilidade de rebanho (EspR, SenR), calculada de acordo com Martin et al. (1992), para uma confiança de 95\%. A SenR e EspR foram estimadas de acordo com Noordhuizen et al. (1997), com o ponto de corte igual a um, significando que o achado de apenas um animal positivo no rebanho já o classificava como rebanho positivo. Calculou-se SenR e EspR de cada propriedade classificada como positiva e, com a média aritmética destas, a SenR e EspR do estrato.

Os valores preditivos positivo e negativo (VPP, VPN) do diagnóstico em rebanhos foram estimados de acordo com Noordhuizen et al. (1997).

\section{Análise dos fatores de risco}

Para o estudo dos fatores de risco associados à soropositividade para $B$. abortus, foi realizada análise univariada por meio da estimativa intervalar da odds ratio (OR), executada com o auxílio do programa Win Episcope 2.0.

\section{RESULTADOS}

O Quadro 2 apresenta os resultados dos testes diagnósticos de triagem e confirmatório em animais, seguidos da classificação das propriedades amostradas nos diferentes municípios do estrato estudado.

Dos 2.376 animais examinados, 274 foram positivos no teste de triagem (AAT). Destes, 157 foram confirmados por meio do teste 2-ME, 18 resultaram inconclusivos e 99 negativos. Dos 210 rebanhos amostrados, 83 foram positivos, quatro inconclusivos e 123 negativos; classificando as propriedades como: positivas, inconclusivas e negativas, respectivamente.

As estimativas das prevalências aparente e real de animais positivos e inconclusivos e das propriedades classificadas como positivas e inconclusivas estão apresentadas no Quadro 3.

O Quadro 4 sumariza os percentuais de PaR, PrR, VPP e VPN.

As variáveis que mostraram associação com a soropositividade para brucelose estão apresentadas no Quadro 5.

\section{DISCUSSÃO}

A inexistência de dados de prevalência divulgados em meios científicos sobre a brucelose bovina no rebanho do Estado de Mato Grosso do Sul (MS) dificulta uma análise comparativa com a obtida neste estudo. No entanto, ao se confrontar a prevalência em animais para a região Centro-Oeste $(6,8 \%)$, e para MS $(6,3 \%)$ 
Quadro 2. Resultado dos testes em série para brucelose bovina; diagnóstico e classificação das propriedades amostradas por município no Estado de Mato Grosso do Sul

\begin{tabular}{|c|c|c|c|c|}
\hline \multirow[t]{2}{*}{ Municípios } & \multirow[t]{2}{*}{$\begin{array}{l}\text { Positivos } \\
\text { na triagem }\end{array}$} & $\begin{array}{c}\text { Teste confirmatório } \\
\text { em animais }\end{array}$ & \multirow[t]{2}{*}{$\begin{array}{l}\text { Rebanhos } \\
\text { amostrados }\end{array}$} & $\begin{array}{c}\text { Classificação das } \\
\text { propriedades }^{a}\end{array}$ \\
\hline & & Positivos Inconclusivos & & Positivas Inconclusivas \\
\hline
\end{tabular}

\begin{tabular}{lc} 
Angélica & 2 \\
Bandeirantes & 10 \\
Caarapó & 11 \\
Campo Grande & 33 \\
Deodápolis & 10 \\
Douradina & 3 \\
Dourados & 22 \\
Fátima do Sul & 1 \\
Glória de Dourados & 4 \\
Itaporã & 1 \\
Ivinhema & 11 \\
Jaraguari & 19 \\
Jateí & 12 \\
Maracaju & 8 \\
Nova Alvorada do Sul & 14 \\
Novo Horizonte do Sul & 10 \\
Ribas do Rio Pardo & 24 \\
Rio Brilhante & 5 \\
Rochedo & 10 \\
Sidrolândia & 33 \\
Terenos & 28 \\
Vicentina & 3 \\
Totais & 274 \\
\hline
\end{tabular}

$\begin{array}{cc}1 & - \\ 8 & 1 \\ 6 & - \\ 15 & - \\ 8 & - \\ 1 & - \\ 14 & 2 \\ 1 & - \\ 2 & - \\ 1 & - \\ 6 & 1 \\ 10 & 2 \\ 4 & - \\ 8 & - \\ 6 & 3 \\ 7 & 1 \\ 19 & - \\ 4 & - \\ 5 & - \\ 18 & 2 \\ 11 & 5 \\ 2 & 1 \\ 157 & 18\end{array}$

$\begin{array}{ccc}6 & 1 & - \\ 8 & 6 & - \\ 8 & 3 & - \\ 18 & 6 & - \\ 9 & 3 & - \\ 2 & 1 & - \\ 17 & 7 & 1 \\ 5 & 1 & - \\ 9 & 2 & - \\ 6 & 1 & - \\ 14 & 4 & - \\ 10 & 7 & - \\ 7 & 2 & - \\ 10 & 5 & - \\ 8 & 4 & 1 \\ 10 & 4 & - \\ 18 & 7 & - \\ 6 & 2 & - \\ 6 & 2 & - \\ 17 & 8 & - \\ 11 & 6 & 1 \\ 5 & 1 & 1 \\ 210 & 83 & \end{array}$

a Ponto de corte igual a 1 .

Quadro 3. Prevalências aparente e real obtidas a partir do teste confirmatório para brucelose em 2.376 bovinos e 210 propriedades amostradas no Estado de Mato Grosso do Sul

\begin{tabular}{|c|c|c|c|c|c|c|}
\hline \multirow[t]{2}{*}{ Classificação } & \multicolumn{3}{|c|}{ Prevalência aparente } & \multicolumn{3}{|c|}{ Prevalência real } \\
\hline & $\% \quad \mathrm{I}$ & IC inferior & IC superior & $\%$ & IC inferior & IC superior \\
\hline \multicolumn{7}{|l|}{ Animais } \\
\hline Positivos $(\mathrm{n}=157)$ & 6,6 & 5,6 & 7,6 & $5,6^{\mathrm{a}}$ & 5,1 & 6,2 \\
\hline Inconclusivos $(\mathrm{n}=18)$ & 0,8 & 0,4 & 1,2 & 0,0 & 0,0 & 0,2 \\
\hline \multicolumn{7}{|c|}{ Propriedades } \\
\hline Positivas $(\mathrm{n}=83)$ & 39,5 & 32,9 & 46,1 & $37,3^{b}$ & 33,3 & 41,4 \\
\hline Inconclusivas $(n=4)$ & 1,9 & 0,0 & 0,0 & 0,0 & 0,0 & 1,1 \\
\hline
\end{tabular}

Quadro 4. Valores preditivos e prevalências, aparente e real, de brucelose bovina nas propriedades estudadas no Estado de Mato Grosso do Sul

\begin{tabular}{lc}
\hline Rebanhos positivos $(\mathrm{n}=83)$ & $\%$ \\
\hline Prevalência aparente & 39,5 \\
Prevalência real & 37,3 \\
Valor preditivo positivo & 72,2 \\
Valor preditivo negativo & 82,5
\end{tabular}

(Paulin \& Ferreira Neto 2003), observa-se que a prevalência aparente obtida (6,6\%) (Quadro 3) quase se equipara a essas, diferindo dos dados oficiais, publicados nos Boletins de Defesa Sanitária Animal, os quais mostram que a prevalência média de animais positivos no Brasil se manteve entre 4 e $5 \%$ no período de 19881998 (Brasil 2003, Paulin \& Ferreira Neto 2003).
A prevalência real de 37,3\% de brucelose no rebanho bovino do Estrato 1 de MS (Quadros 3 e 4) nas 210 propriedades amostradas é inferior à observada no Estado de Mato Grosso (MT) (INDEA 2004), que ao utilizar metodologia semelhante encontrou $42,2 \%$. A soroprevalência para brucelose em rebanhos estimada neste estudo é, também, bastante inferior à observada por Tocantins (2000) no baixo Pantanal de Cáceres, MT, onde a doença estava presente no rebanho bovino em cerca de $82 \%$ das propriedades estudadas. De forma semelhante, quando se analisa a prevalência real em animais $(5,6 \%)$ (Quadro 3 ) observa-se que a estimativa (INDEA 2004) para o MT é superior (10,25\%).

Valores preditivos de rebanho são análogos aos valores preditivos do teste, aplicado individualmente. A sensibilidade de rebanho é a probabilidade que um rebanho positivo seja classificado como tal por um teste positivo de seus indivíduos e 
Quadro 5. Fatores de risco para a brucelose nos rebanhos bovinos estudados no Estado de Mato Grosso do Sul, com os respectivos valores das odds ratio (OR)

\begin{tabular}{|c|c|c|c|c|c|c|}
\hline Variáveis & Descrição & Positivos & Negativos & OR & $\mathrm{IC} \% \mathrm{a}$ inferior & IC\% superior \\
\hline \multirow[t]{6}{*}{ Exploração } & Corte & 40 & 38 & & & \\
\hline & Leite & 25 & 67 & 2,82 & 1,49 & 5,34 \\
\hline & Corte & 40 & 38 & & & \\
\hline & Mista & 18 & 18 & 1,05 & 0,48 & 2,32 \\
\hline & Leite & 25 & 67 & & & \\
\hline & Mista & 18 & 18 & 0,37 & 0,17 & 0,83 \\
\hline \multirow[t]{2}{*}{ Raça } & Zebu & 42 & 36 & & & \\
\hline & Mestiço & 29 & 65 & 2,62 & 1,40 & 4,88 \\
\hline \multirow[t]{2}{*}{ Aborto } & Sim & 33 & 33 & & & \\
\hline & Não & 48 & 88 & 1,83 & 1,01 & 3,33 \\
\hline \multirow[t]{2}{*}{ Ingresso de animais } & Compra animais & 41 & 42 & & & \\
\hline & Não compra animais & 48 & 75 & 1,53 & 0,87 & 2,68 \\
\hline \multirow[t]{2}{*}{ Vacinação } & Não vacina & 15 & 20 & & & \\
\hline & Vacina & 68 & 103 & 1,14 & 0,54 & 2,37 \\
\hline \multirow[t]{2}{*}{ Fonte de contaminação } & Existe área alagadiça & 62 & 91 & & & \\
\hline & Não existe & 21 & 32 & 1,04 & 0,55 & 1,97 \\
\hline \multirow[t]{2}{*}{ Reprodução } & Touro & 72 & 111 & & & \\
\hline & Inseminação artificial e tourc & ro 9 & 10 & 0,72 & 0,28 & 1,86 \\
\hline
\end{tabular}

especificidade de rebanho é a probabilidade que um rebanho negativo seja da mesma forma classificado como negativo por resultados negativos obtidos de seus indivíduos (Martin et al. 1992). Os fatores que afetam a prevalência real, a sensibilidade e especificidade de rebanho também afetam os valores preditivos de rebanho (Martin et al.1992). Neste contexto, o valor preditivo positivo de rebanho $(72,2 \%$, Quadro 4$)$ significa a probabilidade pré-teste que o rebanho seja positivo e esta probabilidade pode variar de acordo com o tipo e o tamanho de rebanho, a localização geográfica ou outros fatores de risco (Christensen \& Gardner 2000).

Conforme Martin et al. (1992), a sensibilidade de rebanho e a especificidade de rebanho são dependentes da sensibilidade e da especificidade do teste individual, do número de animais testados, da prevalência real e do valor do ponto de corte $(=1$, Quadro 2) e da variação na sensibilidade, especificidade e prevalência real entre os rebanhos (Quadros 3 e 4).

A variação na distribuição da brucelose nos diferentes municípios e nas propriedades (Quadro 2) pode estar relacionada com os diversos fatores, como fonte de aquisição de animais, práticas de manejo e clima (Luna-Martínez \& Mejía-Terán 2002, Rosales et al. 2002).

Neste estudo foi observado que, no grupo dos animais positivos, há mais chances de ocorrência da infecção por Brucella abortus em decorrência do predomínio dos seguintes fatores de risco: sistema de exploração de corte $(\mathrm{OR}=2,82$, IC 95\% $=1,49$ $5,34)$, raça Zebu $(O R=2,62$, IC $95 \%=1,40-4,88)$ e aborto $(O R=$ 1,83, IC 95\% = 1,01- 3,33) (Quadro 5).

A constatação de que, dentre os animais positivos para a brucelose, os provenientes de rebanhos de corte têm 2,82 vezes mais chances de apresentarem a doença que os de rebanho leiteiro está de acordo com Campero et al. (2003), que, ao estudarem, na Argentina, fetos abortados provenientes dos dois sistemas de exploração, isolaram o agente principalmente nos re- banhos de corte. Da mesma forma, Poester et al. (2002), no Brasil, afirmam que a brucelose é altamente prevalente em rebanhos de corte.

A predominância de animais positivos em rebanhos de corte e da raça Zebu (OR $=2,62$, IC $95 \%=1,40-4,88)$ pode ser justificada pelo tipo de exploração (recria e engorda), que pressupõe ingresso, muitas vezes, indiscriminado de animais (Poester et al. 2002), embora, nesse estudo, essa variável não tenha identificado relação positiva com a ocorrência da infecção $(O R=$ 1,53 , IC 95\% = 0,87-4,8). O recomendado é que, na compra de animais, esses sejam testados na origem e retestados logo após a entrada no quarentenário, o que diminui as chances de introdução da infecção em propriedades livres (Sanderson et al. 2000, Luna-Martínez \& Mejía-Terán 2002).

A presença do aborto como fator de risco $(\mathrm{OR}=1,83$, IC $95 \%$ $=1,01-3,33$ ) neste estrato representa uma importante fonte de infecção, pois os animais podem se infectar por meio dos fetos abortados, envoltórios fetais, descargas vaginais (Brautigam Rivera 1997, Luna-Martínez \& Mejía-Terán 2002, Campos et al. 2003).

A não utilização de vacina $(\mathrm{OR}=1,14$, IC $95 \%=0,54-2,37)$, não foi identificada como possível fator de risco associado à soropositividade para B. abortus. Entretanto, cabe salientar que o período de realização da entrevista coincidiu com a exigência formal por parte da IAGRO quanto à vacinação antibrucelose, 0 que pode ter influenciado no nível de resposta, apesar da pergunta ter sido direcionada ao uso da vacina antes da instalação do PNCEBT.

A existência de áreas alagadiças nas propriedades não foi constatada como fator de risco associado à soroprevalência da brucelose $(\mathrm{OR}=1,04$, IC 95\% = 0,55-1,97), discordando de Acha \& Szyfres (1986), Paulin \& Ferreira Neto (2003), que relatam que a presença de rios e açudes dividindo lotes de animais de propriedades vizinhas, sombreamento, umidade e baixas tem- 
peraturas favorecem a sobrevivência do agente no ambiente, aumentando o risco de transmissão.

O uso somente de monta natural nas propriedades não constituiu um risco entre os animais soropositivos, pois resultou em relação $<1$ (OR 0,72), quando comparado com a prática da inseminação artificial seguida de repasse com touros. Essa constatação está de acordo com Campero (1993), o qual considera que, embora os touros também possam participar da transmissão da brucelose, pois eliminam a brucela por meio do sêmen, não possuem papel relevante, porque as fêmeas apresentam as defesas naturais na vagina. Entretanto, assumem importância quando da inseminação artificial, pois, nesse caso, o sêmen é depositado diretamente no útero, escapando das barreiras naturais. Os processos de preparação, conservação e descongelamento do sêmen não comprometem a sobrevivência de brucelas, todavia, se adotados todos os protocolos de triagem clínica e sorológica, pelas centrais de inseminação, a possibilidade de obter sêmen de touro infectado é mínima (Acha \& Szyfres 1986, Campos et al. 2003, Paulin \& Ferreira Neto 2003).

Nas propriedades classificadas como positivas, cabe ao sanitarista orientar no sentido de evitar a manutenção da brucelose nos rebanhos já infectados, pois se sabe que essa se deve, principalmente, às falhas de manejo, no que se refere ao atendimento a partos e destino de restos de abortos (Luna-Martínez \& MejíaTerán 2002).

Com base nos resultados obtidos, conclui-se que a prevalência de brucelose no estrato estudado não variou de forma relevante em relação aos achados de levantamentos anteriores, e que os principais fatores de risco que se associam à enfermidade, como a exploração de corte, a raça Zebu e a presença de aborto, podem ser adequados para dirigir o programa de controle da enfermidade.

Agradecimentos.- À equipe de médicos-veterinários, agentes agropecuários e secretárias dos escritórios locais da IAGRO dos 22 municípios, que não mediram esforços no desempenho das atividades durante a operacionalização dos trabalhos de campo.

\section{REFERÊNCIAS}

Acha P.N. \& Szyfres B. 1986. Zoonosis y enfermidades transmissibles comunes al hombre y a los animales. $2^{\mathrm{a}}$ ed. Organización Panamericana de la Salud, Washington, DC. 989p.

Almeida R.F.C. 2001. Diagnóstico da brucelose e tuberculose bovina. Monografia de Graduação, Universidade Federal de Mato Grosso do Sul, Campo Grande, MS. 30p.

Brasil 2001. Instrução Normativa /SDA no. 2 de 10.1.2001. Diário Oficial da União, 4 jun. 2001. Seção 1, p.26-31. Secretaria de Defesa Animal, Ministério da Agricultura, Pecuária e Abastecimento, Brasília. http:/ www.agricultura.gov.br/das/dda/programa html.

Brasil 2003. Manual Técnico do Programa Nacional de Controle e Erradicação da Brucelose e da Tuberculose PNCEBT. Ministério da Agricultura, Pecuária e Abastecimento, Brasília. 130p.

Brautigam Rivera F.E. 1997. Notas de brucelose. Curso de Imunodiagnóstico em Medicina Veterinária. Anais I Curso de Imunodiagnóstico em Medicina Veterinária, Campo Grande, MS. 40p.

Campaña R.C. Gotardo D.J. \& Ishizuka, M.M. 2003. Epidemiologia e profilaxia da brucelose bovina e bubalina. Coordenadoria de Defesa Agropecuária de São Paulo. http://www.cda.sp.gov.br/DocEst/Docs/bru/ info_doc_bru1.htm.
Campero C.M. 1993. Brucelosis en toros: uma revisión. Vet. Med., B.Aires, 74:8-14.

Campero C.M., Moore D.P., Odeon A.C., Cipolla A.L. \& Odriozola E. 2003. Aetiology of bovine abortion in Argentina. Vet. Res. Commun. 27:259269.

Campos A.C.P., Freneau G.E., Acypreste C.S., Dias-Filho F.C., Bueno V.F.F., Souza J.P. \& Resende L.C. 2003. Brucelose Bovina: prevalência de anticorpos anti-Brucella abortus em reprodutores bovinos na microrregião de Goiânia. Ciência Anim. Bras. 4(2):125-129.

Cavalléro J.C.M. 1998. Enfermidades causadoras de aborto: brucelose. In: Lemos, R.A.A. (ed.) Principais Enfermidades de Bovinos de Corte do Mato Grosso do Sul: reconhecimento e diagnóstico. Universidade Federal de Mato Grosso do Sul, Campo Grande, MS. 536p.

Christensen J. \& Gardner I.A. 2000. Herd-level interpretation of test results for epidemiologic studies of animal diseases. Prev. Vet. Med. 45:83-106.

Crawford P.R., Huber J.D. \& Adams B.S. 1990. Epidemiology and surveillance, p.139-141. In: Nielsen K. \& Duncan J.R. (ed.) Animal Brucellosis. CRC Press, Boca Raton, USA.

Davies G. 1971. The rose bengal test. Vet. Rec. 88(17):447-449.

IAGRO 2003. Informe da Campanha de Vacinação contra a Febre Aftosa. Consolidado das regiões de Planalto e Pantanal, etapa novembro de 2003. Agência Estadual de Defesa Sanitária Animal e Vegetal de Mato Grosso do Sul, Campo Grande, MS.

IBGE 2002. Resolução PR-051, de 31.7.1989. Divisão de Pesquisa no Estado de Mato Grosso do Sul, Setor de Documentação e Disseminação de Informações, Instituto Brasileiro de Geografia e Estatística, Campo Grande, MS.

INDEA 2004. Instituto de Defesa Agropecuária do Estado de Mato Grosso, Cuiabá, MT. http://www.indea.mt.gov.br/htlm/index.php

Luna-Martínez J.E. \& Mejía-Terán C. 2002. Brucellosis in México: current status and trends. Vet. Microbiol. 90:19-30.

Martin S.W., Meek A.H. \& Willeberg P. 1987. Veterinary Epidemiology: principles and methods. Iowa State University Press, Ames. 343p.

Martin S.W., Shoukri M. \& Thorburn M.A.1992. Evaluating the health status of herds based on tests applied to individuals. Prev. Vet. Med. 14:33-43.

Noordhuizen J.P.T.M., Frankena K., Van Der Hoofd C.M. \& Graaf E.A.M. 1997. Application of Quantitative Methods in Veterinary Epidemiology. Wageningen Pers, Wageningen, The Netherlands. 445p.

OIE 1987. Brucelosis bovina, ovina y caprina. Office International des Épizooties, Sér.Téc. 6:282.

Paulin L.M. \& Ferreira Neto J.S. 2003. O Combate à Brucelose Bovina: situação brasileira. $1^{\text {a }}$ ed. Funep, Jaboticabal, SP. 154p.

Pellegrin A.O., Leite R.M.H., Guimarães P.H.S., Lage A.P. \& Leite R.C. 1999. Prevalência de brucelose bovina no Pantanal Matogrossense. $26^{\circ}$ Congr. Bras. Med. Vet., Campo Grande, MS. (Resumos em CDRom)

Poester F.P., Gonçalves V.S.P. \& Lage A.P. 2002. Brucellosis in Brazil. Vet. Microbiol. 90:55-62.

Rosales J.F.M., Evangelista T.B.R., Bernal R.S. \& Gómez M.F.M. 2002. Seroprevalencia y factores de riesgo asociados a la brucelosis bovina em hatos lecheros de Tijuana, Baja Califórnia. Tec. Pecu. Méx. 40(3):243-249.

Sanderson M.W., Dargatz D.A. \& Garry F.B. 2000. Biosecurity practices of beef cow-calf procedures. J. Am. Vet. Med. Assoc. 2:217.

Thrusfield M. 1984. Veterinary Epidemiology. 2nd ed. Butterworth, London. 480p.

Tocantins S. 2000. Distribuição espacial da brucelose no gado bovino no Pantanal de Mato Grosso e relação com fatores ambientais. Tese de Mestrado, Curso de Biologia (Ecologia). http//:www.cpap.embrapa.br/ agencia/congresso/8-25.cintra.htm

Vasconcellos S.A., Ito F.H. \& Cortes J.A. 1987. Bases para prevenção da brucelose bovina. Comun. Cient. Fac. Med. Vet. Zootec. Univ. São Paulo, 11:25-36. 